\title{
Prevalence and distribution of Schistosoma haematobium infection among school children living in southwestern shores of Lake Malawi
}

\author{
Sekeleghe Kayuni ${ }^{1,2}$, Rosanna Peeling ${ }^{3}$, Peter Makaula ${ }^{4}$ \\ 1. Department of Parasitology, Liverpool School of Tropical Medicine, Liverpool, United Kingdom \\ 2. Medical Aid Society of Malawi (MASM) Medic Clinics, Blantyre, Malawi \\ 3. Department of Clinical Research, London School of Hygiene \& Tropical Medicine, London, United Kingdom \\ 4. Research for Health, Environment and Development (RHED) Malawi, Mangochi, Malawi
}

Correspondence: Dr Sekeleghe Kayuni (sekekayuni@live.com)

\section{Background}

\section{Abstract}

The prevalence of Schistosoma haematobium infection has been shown to be about $23.7 \%$ among children living in the lakeshore areas of Malawi, with reinfection rates of about $30 \%$ to $40 \%$. This study aimed to determine the current prevalence and distribution of S. haematobium infection in school children along the southwestern shores of Lake Malawi and examine the control interventions present in the area.

Methods

This prospective cross-sectional study was conducted in primary schools. School children were enrolled, demographic data were collected, and urine samples were submitted for analysis on macrohaematuria, microhaematuria, and S. haematobium eggs. A questionnaire was administered to 3 health facilities on diagnosis and treatment of schistosomiasis, as well as the control interventions against it.

Results

Four hundred children (174 males and 226 females) were enrolled from 7 primary schools. Mean participant age was 9.57 years (range 7 to 12 years). Fifty children $(12.5 \%)$ had $S$. haematobium eggs detected in their urine, with the mean egg count being 15/10 $\mathrm{mL}$. The highest infection intensity category ( $\geq 50 \mathrm{eggs} / 10 \mathrm{~mL}$ ) was seen in 10 children (2.5\%). Prevalence varied significantly between the schools, with rates ranging from $0 \%$ to $20 \%$. Schools with higher prevalence rates were located farther away from the nearest public hospital that provides treatment free of charge. Prevalence correlated with previous history of mass chemotherapy in schools.

Mass chemotherapy, health education, and improved water supply and sanitation were some of the interventions that contributed to lower prevalence rates in some areas.

\section{Conclusions}

Schistosomiasis prevalence around southwestern Lake Malawi was lower than previously reported, owing to control interventions focusing on health education, improved water supply, sanitation, and mass chemotherapy. Consistent and uniform interventions can reduce prevalence further and sustain control. As prevalence falls, diagnostics can identify high transmission areas, monitor disease trends, and guide evidence-based control strategies.

\section{Introduction}

Schistosomiasis remains one of the most prevalent parasitic infections in the world and is endemic in 76 countries and territories. ${ }^{1}$ It continues to be a public health problem in the developing world, causing major morbidity and mortality, with over 652 million people estimated to be at risk of the disease, and over 200 million infected; $85 \%$ of the disease burden is in Africa.

Schistosomiasis is endemic in Malawi, and the disease is widely distributed in all regions, causing considerable morbidity. ${ }^{2}$ Malawi is located south of equator, with $20 \%$ of Malawi's land surface being occupied by Lake Malawi, the third largest lake in Africa. The Lake is a renowned tourist destination, which contributes significantly to the country's economy and also supports the fishing industry, agriculture, and national hydroelectric power generation. Mangochi District, which has an estimated population of 1 million people, harbours the lower tip of Lake Malawi.

Genitourinary schistosomiasis, caused by Schistosoma baematobium, is prevalent in Malawi's Southern Region, and intestinal schistosomiasis, caused by Schistosoma mansoni, is endemic to the country's central plateau, its Northern Region, and to the Lower Shire Valley. All water bodies in Malawi are considered to be potential transmission sites for the disease, but this is particularly true for Lake Malawi and the Shire River. ${ }^{3,4}$ Eighty percent of Malawi's population was estimated to be at risk of schistosomiasis in the 1990s. ${ }^{4}$
Schistosoma baematobium is more prevalent in Malawi than $S$. mansoni, and therefore more research has been devoted to $S$. haematobium.

Studies conducted in the 1990s and early 2000s, along the southern shores of Lake Malawi (Mangochi District), showed high $S$. haematobium infection prevalence rates-ranging from over $60 \%$ up to $100 \%$ in some communities. ${ }^{5}$ A 1996 study that assessed resident expatriates in Malawi who had visited the lake found a prevalence of $33 \% .{ }^{6}$ Although the 2002 National Survey on Schistosomiasis and Soil-transmitted Helminths found a national schistosomiasis prevalence of $6.9 \%,{ }^{7}$ and a 2006 Blantyre study found a prevalence of $10.4 \%$ among school children, ${ }^{8}$ high prevalence rates persist in communities along Lake Malawi, especially along the southern part. A Nankumba Peninsula study among school children in lakeshore villages found a schistosomiasis prevalence of $36.5 \%$ in 2003 , and $23.7 \%$ in 2007 , with reinfection rates of $30 \%$ to $40 \%$, a year after treatment. ${ }^{9}$

The Malawi Ministry of Health carries out several schistosomiasis control interventions, namely health awareness and education, mass drug administration (MDA) to school-aged children in affected areas, and chemotherapy to symptomatic people at health facilities. ${ }^{10}$ Since no epidemiologic data from after 2007 are available for the southwestern shores of Lake Malawi, this study was set up to investigate the current prevalence and distribution of schistosomiasis and assesses the ongoing control interventions in the area. 


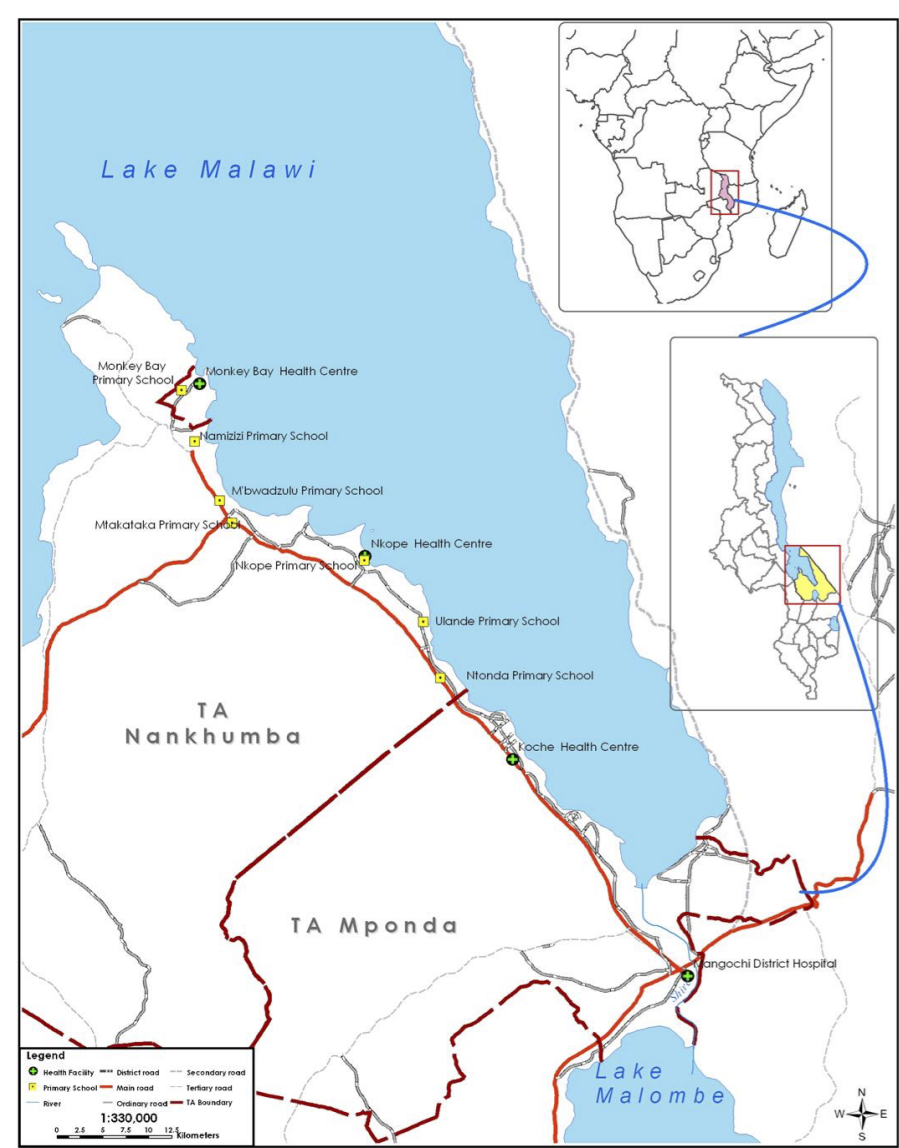

Figure 1: Map of the study area along the Lake Malawi shoreline in Mangochi District

\section{Methods}

\section{Study area and population}

The study area was the southwestern shoreline of Lake Malawi in Traditional Authority (T/A) Nankumba of Mangochi District (Figure 1). The study population was made up of primary school children living in the area. With the high school enrolment rate in Malawi of over $80 \%$, they serve as a better representation of the children living in the area and are a more easily accessible population. The study period was from 1st June 2012 to 31st August 2012, and data collection was conducted from 12th to 22nd June 2012, just before the end of the 2011-2012 academic year.

\section{Study design and sample size}

This was a prospective cross-sectional study among children randomly selected from schools in the study area. All primary schools were eligible for the study and a list of the schools was obtained from the office of District Education Manager (DEM) in Mangochi.

During the study period, the Mangochi District Health Office (DHO) was conducting a campaign of MDA with praziquantel to children in all primary schools in the district. Only primary schools that had not yet undergone MDA were included in the random selection process.

The study initially targeted children from the third year of school (class 3), aiming for a mean age range between 9 and 10 years. According to the World Health Organization (WHO), this age group consistently has highest schistosomiasis prevalence, transmission rate, and infection intensity and serves as a general representative of the community. ${ }^{11}$

A required sample size of 368 children was estimated to measure the current prevalence, taking into account an expected prevalence of $40 \%$, a precision of $5 \%$, and a $95 \%$ confidence interval. ${ }^{12-14}$ The study targeted a minimum of 50 children per school. When the number of children in class 3 was not adequate, children from next class were enrolled.

\section{Data collection}

The selected schools were visited on two consecutive days. On the first day, school authorities, teachers, and children were briefed on the study outline. Thereafter, children were randomly selected and provided with information leaflets and consent forms, which were written in Chichewa, for their parents or guardians. School details were collected from the head teachers on this day.

On the second day, the study team collected data from the children with signed consent forms. The data included demographic details and information on the symptoms and diseases experienced in the preceding month, collected through responses to a questionnaire administered by the teachers. This questionnaire was generating with the guidance of WHO guidelines and previous similar studies. ${ }^{7,11}$

\section{Specimen collection and parasitological analysis}

The children provided fresh urine samples which were analysed for macrohaematuria by visual examination with a urine colour chart, developed in Zanzibar and used in similar studies, ${ }^{15}$ and microhaematuria by using Mindray U-11 urinalysis reagent strips.

Parasitological analysis was conducted on the school premises using a filtration method. ${ }^{16} \mathrm{~A}$ disinfected filter containing a clean $12-\mu \mathrm{m}$ isopore membrane was set up in readiness for the process. Ten millilitres of well-mixed urine was syringed through the filter, trapping $S$. haematobium eggs. Thereafter, the membrane was removed and put on a microscope slide, ready for examination under a light microscope.

As this was an intention-to-treat study, participants who were infected together with the rest of the school children were given praziquantel from the MDA campaign. A pilot study was carried out at an ineligible primary school to test the protocol prior to the initiation of data collection.

The health facilities close to the sampled schools were enrolled into the study, and a questionnaire was administered to senior medical personnel at each facility to assess the schistosomiasis control interventions in place, the availability of treatment, the diagnostic tests available and carried out, and the number of patients treated.

\section{Data analysis and dissemination}

All data were entered into EpiInfo, then checked for irregularities and cleaned. Thereafter, statistical analysis was done using EpiInfo and Stata/IC version 12 software.

The study results were disseminated to the National Schistosomiasis and Soil-transmitted Helminths Control Programme in the Ministry of Health, the district education manager (DEM) and the district health office (DHO) of Mangochi District.

\section{Ethical considerations}

The study was conducted following ethical approval from the Research Ethics Committee of London School of Hygiene and Tropical Medicine, United Kingdom; as well as the Ministry of Health, through the National Health Sciences Research Committee (NHSRC) of Malawi. 
Table 1: Number of children and and number infected in each village

\begin{tabular}{lccc}
\hline Village & $\begin{array}{l}\text { Number of } \\
\text { children }\end{array}$ & $\begin{array}{l}\text { \% of study } \\
\text { sample }\end{array}$ & $\begin{array}{l}\text { Number of } \\
\text { infected } \\
\text { children }\end{array}$ \\
\hline Chilembwe & 12 & 3.00 & 3 \\
Mang'umbi & 27 & 6.75 & 4 \\
Masanje & 4 & 1.00 & 1 \\
Mdala & 4 & 1.00 & 1 \\
Mwalembe & 2 & 0.50 & 0 \\
Guma & 2 & 0.50 & 0 \\
Chiwalo & 41 & 10.25 & 7 \\
Dzumani & 5 & 1.25 & 1 \\
Chindongo & 12 & 3.00 & 2 \\
Maudzu & 8 & 2.00 & 0 \\
Mpale & 13 & 3.25 & 1 \\
Matuwi & 10 & 2.50 & 5 \\
Mpeta & 2 & 0.50 & 0 \\
Koma & 12 & 3.00 & 0 \\
Chizula & 20 & 5.00 & 4 \\
Nangoma & 33 & 8.25 & 10 \\
Chilombo & 32 & 8.00 & 0 \\
Msumbi & 77 & 19.25 & 4 \\
Mbwadzulu & 28 & 7.00 & 2 \\
Madzedze & 33 & 8.25 & 1 \\
Balamanja & 7 & 1.75 & 0 \\
Malata & 3 & 0.75 & 1 \\
Masasa & 4 & 1.00 & 1 \\
Fisheries & 9 & 2.25 & 2 \\
\hline Total & $\mathbf{4 0 0}$ & $\mathbf{1 0 0}$ & $\mathbf{5 0}$ \\
\hline & & & \\
\hline
\end{tabular}

\section{Results}

\section{Study population}

Sixteen primary schools in the T/A Nankumba area were eligible for the study and 7 schools were randomly selected, namely Mtonda, Ulande, Nkope, Mtakataka Turnoff, Mbwadzulu, Namazizi, and Monkey Bay primary schools (Figure 1). The selected schools were within $5 \mathrm{~km}$ of the Lake Malawi shoreline. Three schools, namely Nkope, Mbwadzulu, and Namazizi, received MDA in the 12 months prior to the study.

A total of 415 children with written consent were enrolled into the study. Fifteen children failed to provide urine samples, and their information was not included in the final data analysis. The number of the children per school ranged from 41 to 84 . These children resided in 24 villages served by the 7 schools (Table 1). There were 174 male children (43.5\%) and 226 female children (56.5\%), with an age

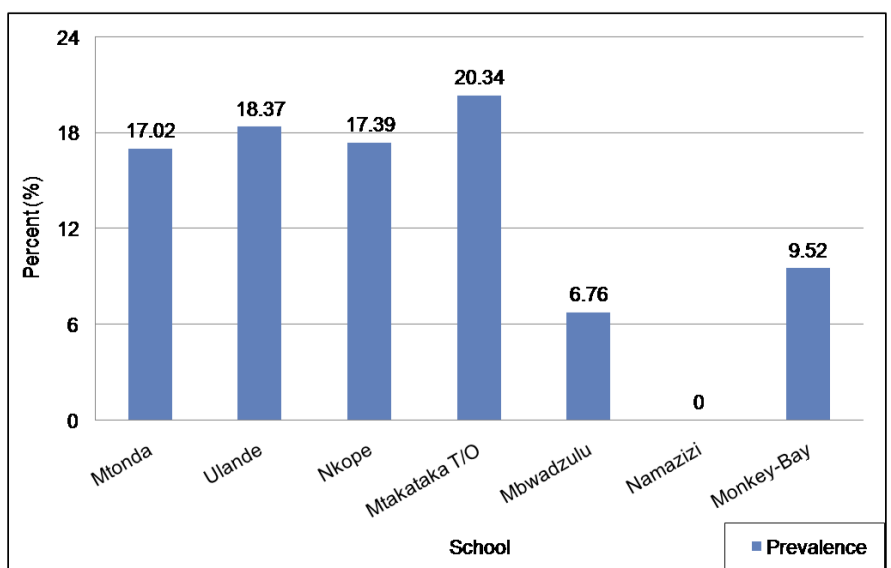

Figure 2: Prevalence of $S$. haematobium infection at each school

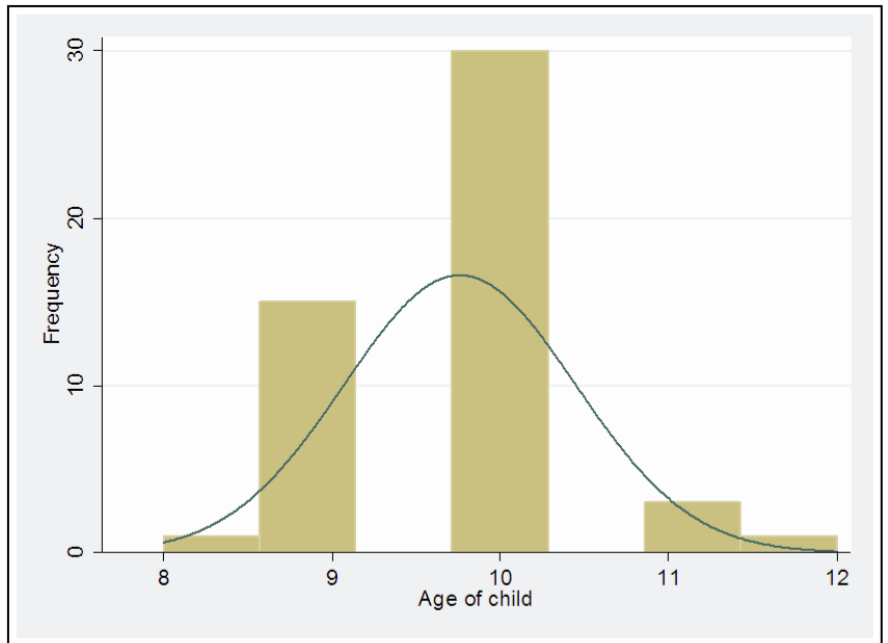

Figure 3: Age distribution of children with $S$. haematobium eggs detected in their urine

range of 7 to 12 years and a mean age of 9.57 years $(95 \%$ confidence interval $[\mathrm{CI}]=9.49$ to 9.66 ).

The mean age for male children was 9.56 years $(95 \% \mathrm{CI}=$ 9.44 to 9.69), while that of female children was 9.58 years $(95 \%$ CI $=9.47$ to 9.69$)$, with no significant difference between the males and females in terms of age $(\mathrm{P}=0.85)$. There were more female children than males in the study sample at all ages, except at 8 and 12 years; and there were more females at all schools, except Mtonda and Monkey Bay.

\section{Prevalence and distribution of schistosomiasis}

Fifty children had $S$. baematobium eggs detected in their urine samples, giving an overall prevalence of $12.5 \%$ (95\% CI $=$ 9.5 to 16.2). The prevalence varied in the schools, ranging from 0\% (Namazizi) to 20.34\% (Mtakataka Turnoff) (Figure 2). The difference in the prevalence among the schools was statistically significant $\left(\right.$ Pearson $\mathrm{X}^{2}=15.51, \mathrm{P}=0.017$ ).

The age of the infected children (children with $S$. haematobium eggs in their urine) ranged from 8 to 12 years, with a mean age of 9.76 years $(95 \% \mathrm{CI}=9.56$ to 9.96$)$. The prevalence rates in children aged $8,9,10,11$, and 12 years were $4 \%$, $10.25 \%, 15.62 \%, 15.79 \%$ and $8.33 \%$, respectively (Figure 3). The variation in prevalence of $S$. baematobium infection with age was not statistically significant (Pearson $\mathrm{X}^{2}=5.26, \mathrm{P}=$ 0.39 ).

The prevalence of infection was $12.1 \%$ among males and $12.9 \%$ among females. Only Mtonda and Monkey Bay schools had a predominance of male children infected (Figure 4). Infection prevalence did not vary significantly by school (Pearson $\left.\mathrm{X}^{2}=0.0523, \mathrm{P}=0.819\right)$. 
Table 2: Urine colour chart scores and urinalysis strip results

\begin{tabular}{|c|c|c|c|c|c|c|c|c|c|c|c|}
\hline \multirow{2}{*}{$\begin{array}{l}\text { S. haematobium } \\
\text { egg count (per10 } \\
\text { mL urine sample) }\end{array}$} & \multicolumn{5}{|c|}{ Urine chart score } & \multicolumn{6}{|c|}{ Urinalysis reagent strip result } \\
\hline & 1 & 2 & 3 & 4 & Total & Neg & Trace & + & ++ & +++ & Total \\
\hline 0 & 333 & 17 & 0 & 0 & 350 & 340 & 3 & 2 & 5 & 0 & 350 \\
\hline 1 to 49 & 17 & 19 & 3 & 3 & 40 & 2 & 4 & 13 & 16 & 5 & 40 \\
\hline$\geq \mathbf{5 0}$ & 2 & 3 & 5 & 0 & 10 & 0 & 0 & 0 & 5 & 5 & 10 \\
\hline Total & 352 & 39 & 8 & 1 & 400 & 342 & 7 & 15 & 26 & 10 & 400 \\
\hline
\end{tabular}

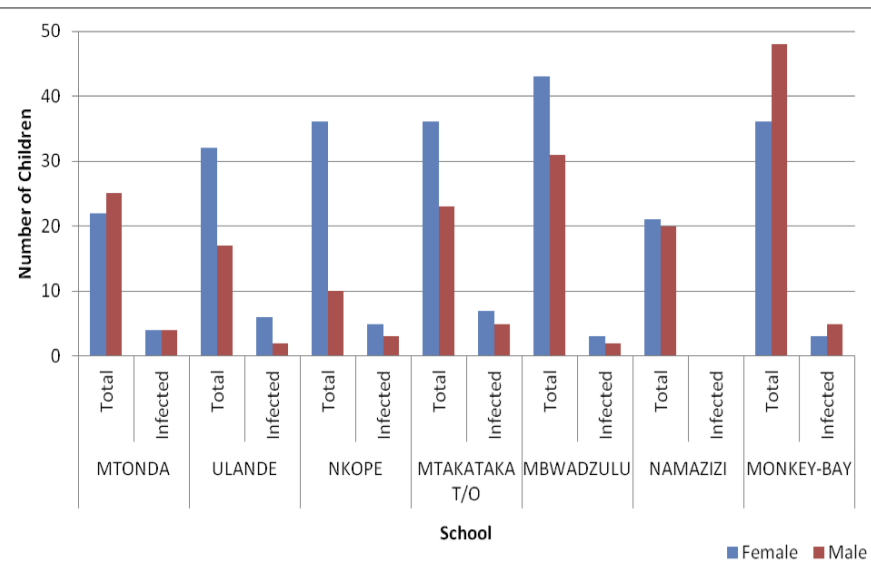

Figure 4: S. haematobium infection frequency by gender and school

With regard to the history of MDA in the previous 12 months, a significantly higher proportion of infected children were found in schools which did not receive MDA (Pearson $\mathrm{X}^{2}=4.82, \mathrm{P}=0.028$ ). All schools except Namazizi and Mtonda had children with more than $50 \mathrm{~S}$. haematobium eggs per $10 \mathrm{~mL}$ of urine. Mtakataka Turnoff School had the highest proportion of infected children (4 out of 12, $33.33 \%$ ) with this high infection itensity.

When analysed for variation by age and gender, the prevalence of $S$. haematobium infection in the children did not vary significantly $(\mathrm{F}=1.57, \mathrm{P}=0.22)$. Similarly, there was no significant variation in $S$. haematobium prevalence when analysed for school and gender as well as school and age (F $=1.02, \mathrm{P}=0.32$; and $\mathrm{F}=0.01, \mathrm{P}=0.91$, respectively).

The mean S. haematobium egg count for the infected children was 15 per $10 \mathrm{~mL}$ of urine. Thirty-three of the 50 children had less than 10 eggs per $10 \mathrm{~mL}$ of urine, and 7 had 10 to 49 eggs. The remaining 10 children $(2.5 \%)$ had 50 or more eggs per $10 \mathrm{~mL}$ of urine and were considered to have the highest intensity of the infection. ${ }^{11}$

Slightly more male children $(2.9 \%)$ had the highest intensity of infection than females $(2.2 \%)$. However, there was no significant difference between the sexes in the proportion of children with highest infection intensity. The age range for the 10 children with the highest infection intensity was 9 to 12 years, with a mean age of 10 years $(95 \% \mathrm{CI}=9.33$ to 10.67). There was a slight positive correlation between $S$. haematobium egg count and age.

With regard to villages, the number of infected children ranged from 0 to 10 (Table 1). Seven of the 24 villages had no infected children. Nangoma Village, which was served by Mbwadzulu and Mtakataka Turnoff schools, had the highest prevalence of infected children (10 [30.30\%] of 33 from the village, and 10 [20\%] of 50 total infected children). The variation in prevalence among the villages was statistically significant (Pearson $\mathrm{X}^{2}=46.97, \mathrm{P}=0.002$ ).

On macrohaematuria using visual examination assisted by the urine colour chart, 333 (95.1\%) of the 350 children http://dx.doi.org/10.4314/mmj.v29i1.4

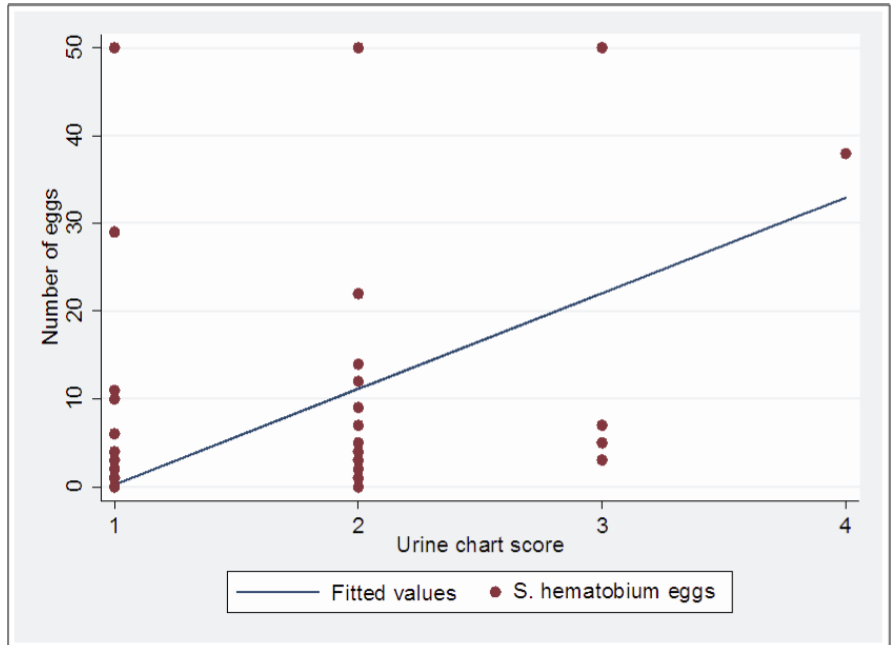

Figure 5: Correlation between $S$. haematobium infection and macrohaematuria

who were not infected had a score of 1 (Table 2). The urine chart scores were strongly correlated with differences in $S$. baematobium egg count (Pearson $\mathrm{X}^{2}=225.21, \mathrm{P}<0.001$ ) (Figure 5). In addition, there was significant variation in odds of $S$. haematobium infection among the different chart scores of the children. Similarly, the results of microhaematuria were strongly correlated with $S$. haematobium egg count (Figure 6). The odds of schistosomiasis infection were also significantly correlated to the different urinalysis results, with higher odds of infection among the positive compared to the negative urinalysis test strip results $(\mathrm{P}<0.001)$, and increasing odds with degree of positivity.

The sensitivity, specificity, and positive and negative predictive values for the urinalysis test compared to the filtration test (which was the gold standard) were 96\%, 97.1\%, 82.8\%, and $99.4 \%$, respectively, with an accuracy of $97 \%$. The likelihood ratio of a positive test ( $\mathrm{LR}+$ ) was 33 , and that of a negative test $(\mathrm{LR}-)$ was 0.04 . These results are considerably high and potentially useful in decision-making on the utility of the test in situations and facilities with limited laboratory infrastructure or expertise.

\section{Information on bealth of the children and schistosomiasis infection}

Cough, abdominal pain, bloody urine (red urine), history of schistosomiasis, worm infestation, and abdominal problems had significantly higher odds of schistosomiasis infection than other symptoms, signs, and other illnesses (Table 3).

The sensitivity, specificity, and positive and negative predictive values for the blood in urine question compared to the filtration test (the gold standard) were $66 \%, 63 \%, 20 \%$, and $93 \%$, respectively, with an accuracy of $63 \%$, LR+ of 1.78, and LR - of 0.54 . Similar results were observed for the history of schistosomiasis question, which had sensitivity, specificity, and positive and negative predictive values of $70 \%, 59 \%, 20 \%$, and $93 \%$, respectively, with an accuracy of $61 \%, \mathrm{LR}+$ of 1.71 , and LR- of 0.51 . 


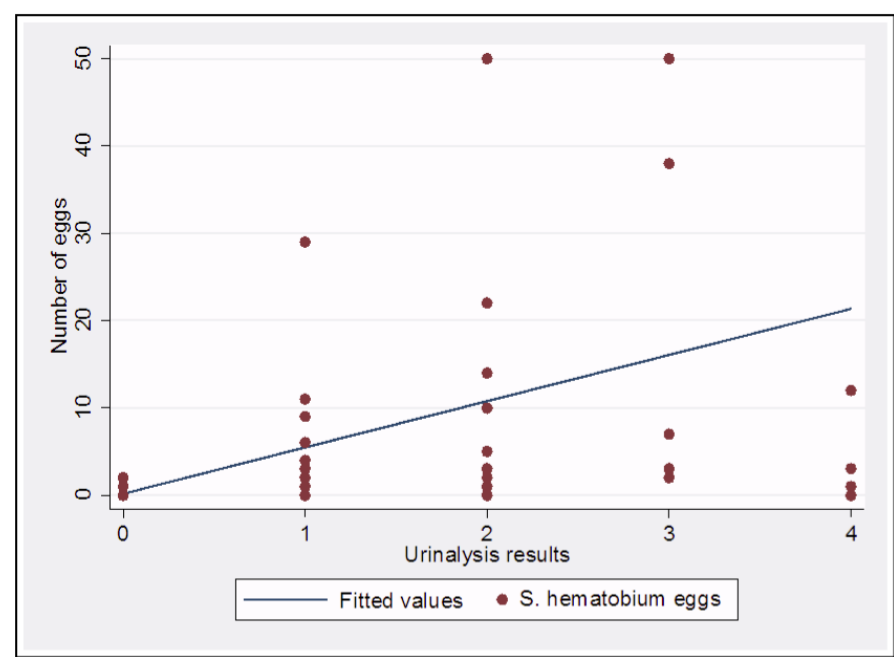

Figure 6: Correlation between S. haematobium infection and microhaematuria

\section{Control interventions for schistosomiasis}

Three health facilities were identified to be close to or serving the selected primary schools. Mtonda, Ulande, and Nkope schools were located in the catchment areas of mission health facilities, owned by religious organisations, while the rest of the schools were within the catchment area of a government-owned facility (Figure 1). All health facilities except Monkey Bay Community Hospital charged a fee to patients for the medical services provided (Table 4).

All facilities used urine microscopy as a diagnostic test for schistosomiasis, except Koche Health Centre, which only used clinical information, owing to limited laboratory expertise. Praziquantel was the drug of choice for schistosomiasis treatment at all health facilities. Only Koche Health Centre had praziquantel for the entire year; Nkope Health Centre and Monkey Bay Community Hospital had the drug only during the last 3 to 6 months of the year, but were otherwise out of praziquantel stock.

Control interventions at the health facilities were health education to patients, provision of toilets and washing areas to admitted patients and their guardians, patient treatment, and MDA. In the community, the interventions were health education, MDA, encouraging construction and use of toilet and bathing areas, encouraging protective wear for fishermen and farmers frequently in contact with water, and avoiding the killing of small molluscivirous fishes that feed on snails. Monkey Bay Community Hospital provided more interventions than the other 2 facilities.

The challenges encountered in implementation of control interventions in the community were lack of drugs for MDA, sandy areas making it difficult to construct toilets and washing and bathing areas, scarcity of water from sources other than the lake, and difficulties with behaviour change (convincing people to stop using the lake for bathing and washing).

The personnel at these facilities highlighted the need to intensify health education efforts and encouragement of communities to build and use toilets and washing and bathing areas, as well as the importance of improving drug availability for patient treatment and consistent MDA.
Table 3: Participant symptoms, signs, and diagnoses in relation to presence of $S$. haematobium eggs in urine

\begin{tabular}{lcc}
\hline $\begin{array}{l}\text { Symptom, sign, or } \\
\text { disease category }\end{array}$ & $\begin{array}{c}\text { Frequency } \\
(\mathbf{N}=\mathbf{4 0 0})\end{array}$ & $\begin{array}{c}\text { P-value } \\
\text { (eggs present) }\end{array}$ \\
\hline Cough & 228 & 0.025 \\
Itching & 133 & 0.84 \\
Headache & 314 & 0.081 \\
Fever & 255 & 0.50 \\
Abdominal pain & 295 & 0.015 \\
Blurred vision & 26 & 0.44 \\
Blood in urine & $\mathbf{1 6 4}$ & $<\mathbf{0 . 0 0 1}$ \\
Blood in stool & 84 & 0.38 \\
Malaria & 302 & 0.66 \\
Diarrhoea & 227 & 0.087 \\
Skin disease & 37 & 0.85 \\
Eye disease & 105 & 0.32 \\
Schistosomiasis & $\mathbf{1 7 7}$ & $<\mathbf{0 . 0 0 1}$ \\
Respiratory disease & 130 & 0.064 \\
Worm infestation & 120 & 0.048 \\
\hline
\end{tabular}

\section{Discussion}

The results of this cross-sectional study indicate that $S$. baematobium infection is prevalent in the area and that transmission is still taking place. This is shown by the presence of infected children (overall prevalence of $12.5 \%$ ) in 6 out of 7 schools investigated.

The prevalence of $S$. haematobium infection was lower than expected, based on results from previous studies. ${ }^{7,9}$ This could be attributed to the comprehensive implementation of control interventions in the health facilities and communities where the children came from. ${ }^{17}$ The interventions are directed toward interrupting the lifecycle of $S$. haematobium, focusing on the human hosts (through awareness and education on reducing contact with contaminated water by encouraging construction and use of toilet, washing, and bathing rooms), parasites (patient diagnosis and treatment, MDA), and intermediate snail hosts (avoiding killing molluscivirous fishes). Much of the environmental control is being implemented through intersectoral collaboration between health authorities (Monkey Bay Community Hospital) and Malawi Department of Fisheries.

Interventions, such as improved clean water supplies, hygiene and sanitation, snail control, and chemotherapy (both preventive and in active infection) are effective control measures in lowering prevalence and intensity of infection. ${ }^{18}$ These are part of the action plans outlined in the new WHO roadmap for combating neglected tropical diseases. ${ }^{19}$ This indicates the commitment by government, partners, and stakeholders in accomplishing goals set at the World Health Assembly in 2001 on reducing morbidity and mortality related to schistosomiasis and soil-transmitted helminthiasis. ${ }^{20}$

In addition, Mangochi District has had an active School Health and Nutrition (SHN) programme, through the Ministry of Education and the Ministry of Health in partnership with various stakeholders. The SHN programme provides annual deworming and schistosomiasis preventive chemotherapy. Some local charity and community-based organisations located along the lake also provide praziquantel regularly to 
Table 4: Health facility information on schistosomiasis control

\begin{tabular}{|c|c|c|c|c|}
\hline \multirow{2}{*}{\multicolumn{2}{|c|}{ Characteristic }} & \multicolumn{3}{|c|}{ Health facility } \\
\hline & & Koche & Nkope & Monkey Bay \\
\hline \multicolumn{2}{|l|}{ Proprietor/owner } & Mission (Catholic) & Mission (Anglican) & Government (public) \\
\hline \multicolumn{2}{|l|}{ Patient service fee } & Paying & Paying & Free of charge \\
\hline \multirow{4}{*}{\multicolumn{2}{|c|}{$\begin{array}{l}\text { Schools served (distance from school to health facility } \\
\text { in } \mathrm{km} \text { ) }\end{array}$}} & \multirow{4}{*}{ Mtonda } & \multirow{3}{*}{ Ulande (7) } & Mtakataka (10) \\
\hline & & & & Mbwadzulu (8) \\
\hline & & & & Namazizi (5) \\
\hline & & & Nkope $(<0.5)$ & Monkey Bay $(<0.5)$ \\
\hline \multirow{4}{*}{\multicolumn{2}{|c|}{$\begin{array}{l}\text { Prevalence of schistosomiasis infection among school } \\
\text { children }\end{array}$}} & \multirow{4}{*}{ Mtonda (17.0) } & \multirow{3}{*}{ Ulande (18.4) } & Mtakataka (20.3) \\
\hline & & & & Mbwadzulu (6.8) \\
\hline & & & & Namazizi $(0.0)$ \\
\hline & & & Nkope (17.4) & Monkey Bay (9.5) \\
\hline Schistosomiasis diagnosti & tests & Clinical & Urine microscopy & Urine microscopy \\
\hline Availability of praziquant & & All year & Last 3 months & Last 6 months \\
\hline \multirow{5}{*}{$\begin{array}{l}\text { Control interventions at } \\
\text { health facility }\end{array}$} & Health education & $\checkmark$ & $\checkmark$ & $\checkmark$ \\
\hline & Proper toilets & & $\checkmark$ & \\
\hline & Proper washing areas & & $\checkmark$ & \\
\hline & Mass drug administration & & & $\checkmark$ \\
\hline & Patient treatment & & & $\checkmark$ \\
\hline \multirow{5}{*}{$\begin{array}{l}\text { Control interventions } \\
\text { in the community }\end{array}$} & Health education & $\checkmark$ & $\checkmark$ & $\checkmark$ \\
\hline & Mass drug administration & $\checkmark$ & & $\checkmark$ \\
\hline & Encourage toilet use & & & $\checkmark$ \\
\hline & Protective wear & & & $\checkmark$ \\
\hline & Avoid killing small fish & & & $\checkmark$ \\
\hline \multirow{4}{*}{$\begin{array}{l}\text { Challenges in } \\
\text { implementing } \\
\text { interventions }\end{array}$} & $\begin{array}{l}\text { No drugs for mass } \\
\text { administration }\end{array}$ & & & $\checkmark$ \\
\hline & Sandy areas & & $\checkmark$ & \\
\hline & Water scarcity & $\checkmark$ & & \\
\hline & Entrenched behaviours & $\checkmark$ & $\checkmark$ & $\checkmark$ \\
\hline \multirow{4}{*}{$\begin{array}{l}\text { Suggestions for control } \\
\text { interventions }\end{array}$} & More health education & $\checkmark$ & $\checkmark$ & $\checkmark$ \\
\hline & Improve drug availability & & & $\checkmark$ \\
\hline & $\begin{array}{l}\text { Mass drug administration } \\
\text { every } 6 \text { months }\end{array}$ & $\checkmark$ & & $\checkmark$ \\
\hline & Encourage toilet use & & $\checkmark$ & \\
\hline
\end{tabular}

schools in their area for treatment and MDA. ${ }^{21}$ This further contributes to the reduction in overall prevalence, as well as a reduction in the proportion of children with higher infection intensity (who serve as reservoirs of infection). Reduced infection rates and intensity allow more children to perform better in class and continue with their education. ${ }^{22,23}$

Infection prevalence rates were diversely distributed in terms of age, gender, and school. The freqeuncy of $S$. haematobium infection among female children was higher than males, which could be due to the higher enrolment of females than males in the selected schools (as well as in Malawi as a whole) ${ }^{24}$ Additionally, most of the children in this study were either 9 or 10 years old (as this was the initial target age range) and few enrolled children fell outside of this range.
However, these factors probably did not undermine the relevance of the results of the study.

Slightly more male children were in the category of highest infection intensity, which has also been noted in other studies. ${ }^{7,8,25}$ It has been suggested that male children have more contact with contaminated water than females because of personality differences, as well as differences in cultural and societal norms of male and female behaviour. However, the possibility remains of similar exposures to the same contaminated water sources.

The prevalence of infection peaked at 10 years of age. This was similar in both sexes, with no significant difference in prevalence between the sexes. This could be attributed to the reduction in exposure to contaminated water sources as 
children grow older, a better understanding of prevention messages, and involvement in other activities that take them away from water bodies. ${ }^{8}$ However, this study had lower numbers of children in the other ages, so caution should be taken when considering such conclusions.

Four schools in the study area (Mtonda, Ulande, Nkope and Mtakataka Turnoff) had higher prevalences of $S$. haematobium infection than the others, indicating the possibility of more transmission taking place in certain areas along the lakeshore. Several factors could explain these differences in prevalence.

Firstly, these schools were located farther away from a free public health facility (Monkey Bay Community Hospital), at distances between 10 and $30 \mathrm{~km}$. This hospital provides comprehensive laboratory and medical services to its patients, as well as praziquantel used for MDA and treating patients with $S$. haematobium infection (although drugs were available for only the half of the year in which data collection for this study took place). Mtonda, Ulande, and Nkope schools are in close proximity to the 2 mission health facilities that charge patients for treatment and other medical services. Many people in the area live on less than US $\$ 2.00$ per day and cannot afford to pay for these services. ${ }^{24,26}$ They are denied access to medication that has a parasitological cure rate of over $85 \%$ and that reduces egg count by $95 \% .{ }^{27}$ This contributes to persistence of the infection in those schools and communities.

Secondly, the mission facilities had limited laboratory services to confirm the diagnosis of schistosomiasis. Treatment decisions therefore relied on clinical information, which has low sensitivity and specificity, ${ }^{7,828,30}$ potentially making the service more expensive because of uninfected people who will be provided schistosomiasis treatment, especially as prevalence declines below $10 \%$. At this lower prevalence, targeted treatment to infected people is more appropriate, given the limited availability of drugs. ${ }^{29}$ The facilities therefore need to improve their laboratory services to diagnose schistosomiasis, using simpler tests that do not require specialised skills, training, or equipment-for example, the WHO urine chart or urinalysis reagent stripsbefore treatment initiation. As the prevalence falls further below 10\%, improved, easy-to-use tests will be useful to target treatment and monitor prevalence trends and effectiveness of infection control measures. ${ }^{29,30}$

Also, there was significant variation in the implementation of control interventions in the study area, despite public health officials doing the work. Monkey Bay Community Hospital provided multifaceted control interventions for infection, unlike the mission facilities (due to limited capacity, support, supervision, and coordination between the mission facilities and the DHO). Communities surrounding the mission facilities therefore did not receive the same comprehensive interventions as those around the public facility.

Additionally, people in communities around Namazizi and Mbwadzulu schools benefitted from the Micronutrient and Health project organised by World Vision International and the National Schistosomiasis Control Programme ${ }^{10}$ from 1998 to 2002, where control interventions included promotion of safe water supplies and sanitation (through constructing boreholes and the provision of cement to every householdsfor building pit latrines), mass chemotherapy with praziquantel and albendazole twice yearly, intensive health education, and iron and vitamin A supplementation. These interventions contributed to a reduction of schistosomiasis prevalence from $78 \%$ in 1998 to $6 \%$ in 2002. This could explain the lower prevalence rates in those schools 10 years later, which suggests the importance of implementing a package of interventions in a consistent way.

Finally, the higher prevalence in the 4 schools could also have resulted from the lack of regular preventive chemotherapy through MDA, which is a safe and lowcost control intervention that has a rapid and sustained impact on infection reduction. ${ }^{18,25}$ Only Nkope School, out of the 4 high-prevalence schools, received MDA in the previous 12 months, highlighting that a lack of treatment leads to uninterrupted transmission, maintenance of an infection reservoir, and a higher incidence of infection. Limited availability of praziquantel in the district could have contributed to inconsistent provision of MDA in the study area.

The children selected from schools in the study sample were drawn up from 24 villages, of which some had significantly higher schistosomiasis prevalence rates. The village that had the highest number of infected children, Nangoma, served Mtakataka Turnof school, which had the highest prevalence among the schools, highlighting the fact that different areas had different transmission rates with varied risk factors for infection. The National Schistosomiasis Control Programme in Malawi has been conducting mapping exercises in some districts in the country, with support from various stakeholders, and this will provide useful information for planning and strategising control interventions. ${ }^{10,31}$ The government can benefit increasingly from the available support from organisations such as Merck, as well as the Schistosomiasis Control Initiative (SCI), who are donating praziquantel tablets to endemic countries to reduce the disease burden in children, following the signing of the London Declaration on neglected tropical diseases on 30th January 2012 in London. . $^{17,26,32}$

This study could not elicit further explanations on the differences in the prevalence of $S$. baematobium infection in some villages. Since selection of the children was done at the schools with regard to their class and age rather than village, much of the conclusions were based on the schools. More research focusing on the villages and communities along the lakeshores will be useful in planning for infection control.

\section{Conclusions}

Schistosomiasis is still prevalent in the study area and transmission continues to take place. Schools that had higher prevalence rates were located farther away from the public hospital in the area that provides free treatment, and these schools did not benefit from MDA in the previous 12 months. The prevalence of $S$. baematobium infection was lower than previously reported, arising from implementation of various control interventions in the area. Consistent interventions and further mapping and monitoring of prevalence trends need to be applied in order to achieve significant and sustained control of schistosomiasis.

\section{Acknowledgements}

We sincerely thank all school head teachers, teachers, and children who took part in the study; as well as the the district health officer, district education manager and their officers for their support. We are also very grateful to $\mathrm{Mr}$ Jemu for all the help, advice, and supplies for the study; to Medical Aid Society of Malawi (MASM) for providing laboratory personnel (Mr Nkhoma), supplies, transport, and accommodation; Mr Mkambeni and Mr Nkhoma for the laboratory expertise; Mr Matekenya of the National Statistics Office (Malawi) for the map of the study area; Dr Q. Bickle and Shaali Ame for their advice on sourcing some study items; Dr L. Chitsulo of the WHO for the filter membranes; and to John Williams, Claire Rogers, and Julie Tucker for sharing their parasitological skills prior to the study. 


\section{Competing interests}

All authors declare that they have no competing interests related to this work.

\section{References}

1. Engels D, Chitsulo L, Montressor A, Savioli L. The Global epidemiological situation of Schistosomiasis and new approaches to control and research. Acta Tropica, 2002; 82:139-46.

2. Teesdale $\mathrm{CH}$, Chitsulo L. Schitosomiasis in Malawi - review. Tropical Medicine Parasitology, 1985; 36:1-6.

3. Schistosomiasis Control Programme. Lakeshore Schistosomiasis Control Project (1997-2001). Lilongwe, Malawi: Ministry of Health and Population; 2002.

4. Poole H, Terlouw DJ, Nauje A., et al. Schistosomiasis in pre-schoolage children and their mothers in Chikhwawa district, Malawi with notes on characterization of schistosomes and snails. Parasites \& Vectors 2014, $7: 153$.

5. National Schistosomiasis Control Programme. Ten year Plan of Action (1991-2000). Lilongwe, Malawi: Ministry of Health and Population; 1991.

6. Cetron M, Chitsulo L, Sullivan JJ, et al. Schistosomiasis in Lake Malawi. The Lancet, 1996; 348:1274-8.

7. Bowie C, Purcell B, Shaba B, Makaula P, Perez M. A National Survey of the Prevalence of Schistosomiasis and soil-transmitted helminths in Malawi. BMC Infectious Diseases, 2004; 4.

8. Kapito-Tembo AP, Mwapasa V, Meshnick SR, et al. Prevalence distribution and risk factors for infection among school children in Blantyre, Malawi. PLoS Neglected Tropical Diseases, 2009; 3.

9. Madsen H, Bloch P, Makaula P, Phiri H, Furu P, Stauffer Jr J. Schistosomiasis in Lake Malawi villages. EcoHealth, 2011.

10. Jemu S. Prevalence and Control of Schistosomiasis in Malawi. Personal Communication with Director of National Schistosomiasis and Soiltransmitted Helminths Control programme, Ministry of Health, Malawi. 13th February 2012.

11. Montressor A, Crompton DWT, Hall A, Bundy DAP, Savioli L. Guidelines for the evaluation of soil-transmitted helminthiasis ans schistosomiasis at community level. In: ORGANISATION, W. H. (ed.). Geneva; 1998.

12. Lwanga S, Lemeshow S. Sample size determination in health studies. A practical manual. In: ORGANISATION, W. H. (ed.). Geneva; 1991.

13. Kirkwood B, Sterne J. Essential Medical Statistics, Malden, Blackwell Science, 2003

14. Bennet S, Woods T, Liyanage W, Smith D. A Simplified general method for Cluster-sample surveys of health in developing countries. World Health Statistics Quarterly 1991; 44, 98-106.

15. Sousa-Figueiredo JC, Basanez M-G, Khamis IS, Garba A., Rollinson D, Stothard,JR. Measuring Morbidity Associated with Urinary Schistosomiasis: Assessing Levels of Excreted Urine Albumin and Urinary Tract Pathologies. PLoS Neglected Tropical Diseases, 2009; 3(10).

16. Cheesbrough M. District Laboratory Practice in Tropical countries Part 1, Cambridge, UK, Cambridge University Press; 2006.
17. Hotez PJ, Molyneux DH, Fenwick A, et al. Control of Neglected Tropical Diseases. The New England Journal of Medicine, 2007; 357:1018-27.

18. Fenwick A, Webster JP. Schistosomiasis: Challenges for control, treatment and drug resistance. Current Opinions in Infectious Diseases, 2006; 19: 577-82

19. WHO. Accelerating work to overcome the global impact of Neglected tropical diseases - A roadmap for implementation. In: WORLD HEALTH ORGANISATION (ed.). Geneva: WHO Press; 2012.

20. WHO. Fifty-fourth World Health Assembly Resolution. WHA54.19. Agenda item 13.3. In: ORGANISATION, W. H. (ed.) World Health Assembly. Geneva: World Health Organisation; 2001.

21. Save The Children (USA). Controlling bilharzia in schools. Successes and lessons learned from Mangochi District, Malawi, September 2008. Washington, DC, 20036: Save the Children (USA).

22. Squire B. Schistosomiasis. In: Gill G, Beeching N. (ed.) Lecture notes on Tropical Medicine. 6th ed. West Sussex: Blackwell Publishing Ltd; 2009.

23. WHO. Prevention and control of schistosomiasis and soil-transmitted heliminthiasis. WHO and UNICEF Joint statement. Geneva: WHO and UNICEF; 2004.

24. NSO \& ICF Macro. Malawi Demographic and Health Survey 2010. Zomba, Malawi: National Statistical Office; 2011.

25. Zhang Y, Toure S, Bosque-Oliva E, et al. Two.year impact of single Praziquantel treatment on infection in the National Control Programme on Schistosomiasis in Burkina Faso. Bulletin of the World Health Organisation, 2008; 86:780-7.

26. Fenwick A. The global burden of neglected tropical diseases. Public Health, 2012; 126:233-6.

27. Taylor M. Global trends in schistosomiasis control. Bulletin of the World Health Organisation [Online]; 2010.

28. Bogoch II, Andrews JR, Dazdie Ephraim RK, Utzinger J. Simple questionnaire and urine reagent strips compared to microscopy for the diagnosis of Schistosoma haematobium in a community in northern Ghana. Tropical Medicine and International Health, 2012; 1-5.

29. Zhou X-N, Xu J, Chen H-G, Wang T-P, et al. Tools to Support Policy Decisions Related to Treatment Strategies and Surveillance of Schistosomiasis Japonica towards Elimination. PLoS Neglected Tropical Diseases, 2011; 5.

30. Solomon AW, Engels D, Bailey RL, et al A Diagnostic Platform for the Integrated Mapping, Monitoring, and Surveillance of Neglected Tropical Diseases: Rationale and target Product Profiles. PLoS Neglected Tropical Diseases, 2012; 6(7): e1746. doi:10.1371/journal.pntd.0001746

31. Brooker S, Kabaterine NB, Gyapong JO, Stothard JR, Utzinger J. Rapid mapping of Schistosomiasis and other Neglected tropical diseases in the context of integrated control programmes in Africa. Parasitology, 2009.

32. WHO. London Declaration on Neglected Tropical Diseases. Uniting to Combat Neglected Tropical Diseases. Royal College of Physicians, London; 2012. 